\title{
Anabases
}

ANABASES Traditions et réceptions de l'Antiquité

$8 \mid 2008$

Varia

\section{Tucidide fra i pensatori della politica}

\section{Paulo Butti de Lima}

\section{OpenEdition}

\section{Journals}

\section{Edizione digitale}

URL: http://journals.openedition.org/anabases/234

DOI: 10.4000/anabases.234

ISSN: 2256-9421

\section{Editore}

E.R.A.S.M.E.

\section{Edizione cartacea}

Data di pubblicazione: 1 ottobre 2008

ISSN: 1774-4296

\section{Notizia bibliografica digitale}

Paulo Butti de Lima, «Tucidide fra i pensatori della politica », Anabases [Online], 8 | 2008, Messo online il 01 juillet 2011, consultato il 20 octobre 2019. URL : http://journals.openedition.org/anabases/234 ; DOI : 10.4000/anabases.234

Questo documento è stato generato automaticamente il 20 ottobre 2019

(c) Anabases 


\title{
Tucidide fra i pensatori della politica
}

\author{
Paulo Butti de Lima
}

1 Una nuova rappresentazione di Tucidide si afferma in Germania negli anni '30 del ventesimo secolo. In vari studi si sottolinea allora la natura di riflessione politica dell'opera dello storico ateniese, in contrapposizione ai suoi aspetti più propriamente storici e narrativi. Lontani gli anni della Grande guerra e gli interessi dei suoi Gelehrten, questa nuova interpretazione annovera Tucidide tra $\mathrm{i}$ " pensatori della politica ${ }^{1}$ ». La moderna parabola ateniese, riscontrata nei percorsi della Germania contemporanea, non lascia più dubbi, sulla disfatta e sulla guerra civile : lo storico di Atene può quindi diventare maestro di politica e di etica statale per chi sente mancare l'ethos alla nazione senza l'Impero. I riferimenti analogici alla situazione attuale o pregressa della Germania sono mediati dai temi universali della politica, tra i quali non tarderà a porsi, in posizione privilegiata, il rapporto tra il capo e la comunità.

2 1. La strada per la nuova rappresentazione di Tucidide viene preparata da Werner Jaeger (1888-1961), allievo di Wilamowitz, che nel 1924 presenta una conferenza a Berlino, per la « Reichsgründungsfeier », sulla " etica statale ai tempi di Platone ». Jaeger vi esprime la nostalgia del Reich e auspica un "vero potere », radicato nella " salute morale e simmetria della comunità popolare (Volksgemeinschaft) ${ }^{2}$ ". Ai temi etici letti attraverso Platone e i suoi contemporanei non resta estraneo Tucidide : qualche anno dopo, nel 1928, un allievo di Jaeger, Wolfgang Schadewaldt (1900-1974), presenta una conferenza sullo storico ateniese, pubblicata l'anno seguente con dedica al maestro (Schadewaldt 1929).

3 Schadewaldt riprende la « questione tucididea » - ovvero, il processo compositivo della narrazione e la sua stratificazione secondo il percorso biografico dell'autore -, alla quale i lavori di Eduard Schwartz e di Max Pohlenz avevano dato nuova vita, alla fine della Prima guerra mondiale ${ }^{3}$. I libri siciliani non furono scritti subito dopo il disastro della spedizione ateniese, ma rappresentano il frutto dell'attività dell'ultimo Tucidide, lo storico che rientra ad Atene dopo gli anni dell'esilio. La catastrofe finale ateniese, nel 404 a.C., è prefigurata nel disastro siciliano del 413 (p. 14). La spedizione in Sicilia 
appariva, già nei momenti della disfatta tedesca nel conflitto mondiale, come la chiave dell'interpretazione dell'opera tucididea e, per analogia, della situazione presente. Un presente, però, che si fa ora più meditato e con radici lontane : non è il « dolore recente dei patrioti per la perdita di una flotta e di un comandante » che rende senso all'opera tucididea, nella sua scrittura finale, ma « il cruccio del figlio del tempo pericleo per lo sperpero della grandezza e del potere della città natale » (p. 21). Erano i sentimenti patriottici a caratterizzare l'ultimo Tucidide, secondo la costruzione classica di Schwartz. Ora invece è soprattutto la riflessione sulla natura della politica e del potere a interessare lo storico rientrato nella città sconfitta.

4 Lontani dai fatti della guerra, ai nuovi e giovani lettori di Tucidide il racconto storico dovrà apparire, in qualche modo, secondario rispetto a fini più elevati. La preoccupazione con la metodologia storica, con l'accertamento dei fatti, la verità come precisione e correttezza - la «historische Richtgkeit»-, caratterizza il "primo» Tucidide, poi convertito alla ricerca del senso vero dell'avvenire storico, la « historische Wahrheit» (p. 39). Resta chiaro che i capitoli iniziali dell'opera, i cosiddetti capitoli «metodologici », appartengono ad una prima fase compositiva, quando Tucidide non è altro che un "historisierende Sophist» (p. 30). Con il disastro siciliano avviene la svolta, e alla narrazione degli avvenimenti si sovrappone la riflessione su di essi, secondo principi universali. Da Pericle alla sconfitta : dopo il 404 Tucidide medita sul disastro della Patria, ne cerca le cause e approda, per mezzo di Alcibiade, alla consapevolezza della politica e alla "sostanza dello Stato ». In quel momento, "der Kriegshistoriker wird notwendig zum Staatsethiker, führt staatsethische Kategorien in seine reale Geschichtsbetrachtung ein ${ }^{4} »$ (p. 18).

5 2. In una recensione critica, pubblicata nel 1930, Felix Jacoby nota il carattere innovatore del breve volume di Schadewaldt ${ }^{5}$. L'anno seguente, interviene Felix Wassermann nei Neue Jahrbücher für Wissenschaft und Jugendbildung ${ }^{6}$, parlando di una "nuova rappresentazione di Tucidide» (Wassermann 1931). In realtà, questa novità consiste, per l'autore, nella stessa questione tucididea, ripresa già con Schwartz e Pohlenz, ora con Schadewaldt. Ma al saggio più recente è riconosciuta l'originalità nel ricostruire la "trasformazione » di Tucidide, una trasformazione che non si restringe ad un cambiamento di giudizio sugli avvenimenti, ma che riguarda la «natura » dello storico. All'interno di questa prospettiva, Wassermann mette in luce il ruolo svolto nella « realtà della vita politica» dai discorsi riportati da Tucidide in quanto mezzo di conoscenza e di azione. L'opera di Tucidide è scritta da uomo politico per uomini politici, che riflettono sulla politica e che prendono parte alle azioni dello Stato (p. 248-249).

6 Nel 1933, Otto Regenbogen (1891-1966) pubblica un saggio denso, pervaso da un tono pessimistico, su «Tucidide come pensatore politico» (Regenbogen 1933). L'influenza delle tesi di Schadewaldt sull'autore si faceva sentire già in precedenza, in conclusione all'analisi di alcuni passi tucididei pubblicata nel 1930 (Regenbogen 1930). Tuttavia, quest'influenza non si trasforma completamente nello sforzo di studiare l'opera da una prospettiva compositivo-biografica ${ }^{7}$. Il tema della "politica» è attuale, ammette Regenbogen nel '33, ma in un senso " più alto » e non secondo la ricerca e affermazione di relazioni immediate con l'attualità. La vera attualità è quella « ungesuchte und unausgesprochene », che risulta dalla pura rappresentazione dell'oggetto (p. 24). Per chi, come Regenbogen, scrive anni dopo la disfatta della Patria, il senso dell'opera storica risiede nella « riflessione » che vi è contenuta. Lo storico si esprime per mezzo 
dei discorsi dei personaggi, che devono essere quindi considerati non soltanto nel loro elemento « realistico » $\mathrm{e}$ " poetico », ma anche di « riflessione ${ }^{8}$ » (p. 26-28).

7 Tucidide, pensatore politico, è «non soltanto il primo storico critico, ma al tempo stesso il primo vero e consapevole pensatore politico della storia spirituale europea ». Nella sua consapevolezza è "figlio dell'età dei sofisti", nella politica, "figlio dell'Attica» (p. 29). "Tucidide scrive come politico per uomini politici» (p.31). Regenbogen individua tre forme in cui Tucidide rappresenta l'uomo in quanto «Träger der politischen Abläufe» (p.36) : come individuo (Temistocle o Pericle, ma anche Antifonte), in quanto tipo (i discorsi contrapposti) e in quanto collettività (Atene e Sparta come "staatliche-politischer Totalitäten »). L'identificazione dello storico con Pericle e il suo patriottismo si realizzano pienamente nel momento del crollo della Patria, e l'ultimo discorso pericleo ne è l'esempio. Tucidide si fa vero "pensatore " nell'esperienza della sconfitta. È allora - e qui risuona la tesi di Schadewaldt - che si vede lo "sviluppo dello storico Tucidide in uno Staatsdenker» (p.57). L'opera dello storico rappresenta quindi l'oggettivazione della sconfitta, di cui il suo moderno lettore accentua più volte il pathos («Leid und Not des Daseins ", "Schmerz und Leid»). Seguendo sempre le tesi dell'allievo di Jaeger, ma più genericamente accostandosi ad uno sviluppo proprio degli studi degli anni dopo la fine della guerra, Regenbogen può concludere: « il senso ultimo dell'opera tucididea si chiama Platone » (p. 58).

Questo Tucidide pensatore risveglia l'interesse di chi si dedica al problema della costruzione di un'etica statale, sulle tracce di un nuovo umanesimo. L'anno seguente alla pubblicazione del suo saggio, Regenbogen rende chiaro il significato programmatico di quel sentimento greco della politica, superiore e distante, con un contributo su "L'antichità e l'educazione politica », pubblicato nei Neue Jahrbücher für Wissenschaft und Jugendbildung (Regenbogen 1934). Per parlare del «nuovo Stato dell'uomo tedesco » l'autore si rivolge alla "politische Sicht des Altertums, die uns heute frommt und nottut ", la politica in senso largo, come per primi i Greci hanno compreso e realizzato (p. 213). Vi troviamo quindi riferimenti alla vita "grigia » del regno asiatico (p. 214) e all'eroismo " nordico » di Alessandro (p. 217 s.), e si rievoca la «Dämonie» eroica dei Greci, cara anche a Schadewaldt. Ancora sulle tracce di Schadewaldt, Regenbogen nota che la prossimità dei contemporanei al mondo ellenico non si rivela per mezzo di facili trasposizioni e analogie : è attraverso la visione antica della politica che si può aprire al senso profondo del nuovo sentire della comunità indivisa, del futuro dello Stato nazional-socialista 9 .

9 3. Tucidide come pensatore politico è ugualmente il titolo scelto da Werner Jaeger (1888-1961), in Paideia, per il capitolo sullo storico ateniese. Il primo volume dell'opera appare, per la prima volta, nel 1934, ma la prefazione è firmata ottobre 1933, lo stesso anno del saggio di Regenbogen ${ }^{10}$. In un altro articolo, pubblicato nel '33 sulla rivista Das Volk im Werden, Jaeger aveva indicato come compito attuale dei Tedeschi «la formazione dell'uomo politico ${ }^{11} »$. L'inclusione di Tucidide tra i pensatori - possibile sempre per mezzo della valorizzazione dei discorsi - non impedisce a Jaeger di rievocare, attraverso le vicende ateniesi, i temi più cari alla storia contemporanea. Guerre antiche e moderne non devono essere considerate dal punto di vista morale : già Tucidide vedeva che non si tratta di « una misera questione di colpa o non colpa », ma del «risultato di un annoso processo incessante, determinato da una necessità superiore » (Jaeger 1934, ed. it. p. 659). Per il più anziano Jaeger, allievo di Wilamowitz, resta sempre presente, di forma tacita, l'equazione Atene-Germania. I « liberatori » - gli 
antichi Spartani, ma, s'intende, anche i moderni Inglesi -, nel loro cinismo, si fanno vedere come "rappresentanti della libertà e del diritto ». Fanno coincidere « le belle parole d'ordine" con il proprio interesse, "sicché non è detto abbiano essi stessi coscienza di dove l'una cosa finisce ed incomincia l'altra ». Agli Ateniesi (e ai Tedeschi) non resta alternativa alla sincerità, che "può apparire brutale, ma è talvolta più simpatica del gergo morale dei "liberatori" » (Jaeger 1934, ed. it. p. 669). Tucidide non si occupa, però, soltanto dei nemici occidentali della Germania, e Jaeger non dimentica ciò che avviene all'Est : gli Ateniesi sono «fieri di non conoscere dispotismo di tipo orientale, ma d'esser invece uno Stato costituzionale moderno » (ed. it. p. 671). Onore nazionale e ragion di Stato, Jaeger riecheggia sempre i temi cari agli intellettuali della Prima guerra. "Niente annessione » è la parola d'ordine periclea, ma la "voce del Tucidide più recente » ci fa capire che non si trovava lì il problema ateniese, bensì nelle divisioni interne alla Patria : « io temo più i nostri errori, che i colpi dei nemici » (ed. it. p. 682).

Jaeger non riprende, così, soltanto gli argomenti più frequentati dalla generazione dei suoi maestri, nel leggere, da parte conservatrice, la politica tedesca per mezzo degli antichi. È anche attento a questioni politiche che assumono ora un valore diverso, e alle quali lo storico ateniese fa sempre da guida ideale :

Il problema del rapporto tra la singola personalità eminente e la comunità politica, che si fa acuto appunto nello Stato della «libertà ed eguaglianza », cioè della moltitudine, è felicemente risolto, per Tucidide, nell'Atene di Pericle.

Dagli insegnamenti della Grecia antica e del suo storico-pensatore, possiamo quindi passare alla norma generale :

La storia insegna che tale soluzione dipende dall'esistenza del capo geniale, che nella democrazia rimane altrettanto rara cosa quanto nelle altre forme politiche, e che anche la democrazia non offre garanzie contro i pericoli della mancanza d'un capo (Jaeger 1934, p. 511 = ed. it. p. 685-686).

Questo insegnamento non sembra, però, sufficiente, per la sensibilità dello studioso $\mathrm{e}$ dei suoi contemporanei. Visto nella prospettiva delle vicende attuali, il modello pericleo di Tucidide appare incompleto. Nelle pagine finali del capitolo su Tucidide in Paideia, e in preparazione a quello platonico, Jaeger si rivolge improvvisamente a Dionigi di Siracusa. Anche in assenza di uno storico come Tucidide, il tiranno siciliano riesce a far lezioni per il presente. Si può certamente riscontrare quanto è mancato alla esperienza politica antica, che «non riuscì a inserire realmente i cittadini nello Stato ». Ma non tutto era negativo in quel mondo, ci fa capire Jaeger con una affermazione che rende senso a questa singolare digressione finale del capitolo tucidideo e che verrà cancellata dalle edizioni successive di Paideia :

Es wird das Ziel des modernen Führerstaates sein müssen, diesen neuen Weg zu finden, der zwischen der demokratisch unterbauten Führerstellung des Perikles und der rein militärisch gestützten Alleinherrschaft des Dionysios hindurchführt (Jaeger 1934, p. 511).

Nel 1936 Jaeger parte per gli Stati Uniti, e in quell'anno appare la seconda edizione di Paideia, per la quale - e di conseguenza per le traduzioni future - sembrava poco adeguato il programma del moderno « Führerstaat ».

4. Curiosamente, sempre nel 1934 viene pubblicata la dissertazione di Ernst Dietzfelbinger, da lui discussa l'anno precedente - con Otto Stählin come Referent -, dal titolo Tucidide come pensatore politico (Dietzfelbinger 1934). L'autore fa in tempo a includere riferimenti ai testi omonimi, e più maturi, di Jaeger e Regenbogen. Ancora 
una volta si segue la strada indicata da Schadewaldt. Per i lettori moderni di Tucidide contano di più "die Gedanken »: per mezzo degli avvenimenti della guerra del Peloponneso lo storico intende sviluppare «le leggi della vita politica» (p. 7). Presto siamo messi di fronte al tema ricorrente : « il grande problema, che governa la storia di ogni popolo... : la relazione tra il singolo e la comunità » (p. 19). Un problema che è caro al giovane autore e al suo tempo, e che sembra a lui essere anche quello di Tucidide. I capitoli metodologici, insegnava Schadewaldt, riguardano la guerra archidamica, mentre la visione matura dello storico gli fa guardare all'insieme degli avvenimenti e a trarne insegnamenti storici e politici. Questi insegnamenti si traducono nei titoli delle parti finali del volume di Dietzfelbinger, come « Der einzelne und der Staat» (p. 107) oppure « Führergedanke » (p. 108). Certamente più originale l'affermazione di Tucidide come educatore popolare: « Thukydides will sein Volk politische erziehen » (p. 124)!

La sovrapposizione dell'interesse pedagogico alla riflessione politica era all'ordine del giorno, e Tucidide doveva prestarsi a tale compito, spesso attribuito a Platone. Se Regenbogen si esercita, nel 1934, sul tema della « educazione politica», lo studioso del pensiero antico, Wilhelm Nestle scrive, sempre nel '34, per una rivista nazionalsocialista, « Tucidide come educatore politico ${ }^{12}$ ». Ancora in quell'anno, il curatore degli Jahrbücher su cui aveva pubblicato Regenbogen, Heinrich Weinstock (1889-1960), pubblica il volume Polis. Der griechische Beitrag zu einer deutschen Bildung heute an Thukydides erläutert, in cui il riferimento al «Führer des Dritten Reiches» (p. 16) è presente insieme ai riferimenti tucididei al « Führer » (p. 67-70). Nel '38 Weinstock cura una traduzione di brani di Tucidide (Der grosse Krieg), accompagnata da didascalie generalizzanti e da una prefazione e postfazione in cui accentua l'importanza della politica (« Ursubstanz ») nella visione dello storico ${ }^{13}$ (Weinstock 1938).

5. Questa stagione di studi su Tucidide come "pensatore della politica» porterà l'attenzione su aspetti diversi dell'opera storica. Vi è una "estensione» del Tucidide politico nel Tucidide militare, sempre letto attraverso i discorsi. Questo ambito militare non è secondario alla politica, osserva il neoumanista Albert Rehm (1871-1949) contro Jaeger (Rehm 1934). Tucidide è certamente un pensatore della politica, ma non si deve dimenticare che "politisches und militärisches Denken hier zusammengehören" (p. 149). La prossimità tra politica e comando militare può essere estranea ai Tedeschi suoi contemporanei, nota l'autore, ma accomuna gli antichi Greci a Federico il Grande ${ }^{14}$. Ai discorsi militari si dedicherà ancora, e lungamente, Otto Luschnat, con un saggio scritto nel '39, ma da lui curato per la pubblicazione negli intervalli delle proprie attività militari (Luschnat 1942).

Sulla particolarità del Tucidide " politico » insiste Hans Bogner (1885-1948) nel $1937^{15}$. «La realtà storica nel nostro senso è per lui il sostrato per la scoperta della verità politica » (p. 16). In questo si distingue Tucidide da Omero e da Erodoto : « Tucidide non vuol essere storico, ma piuttosto qualcosa di diverso, un pensatore politico su base storica " (ibid.). Ancora una volta, l'interesse per Tucidide in quanto pensatore della politica si trasforma nell'attenzione per la guida della nazione e per il rapporto tra il capo e il popolo. Senza più pensare a Pericle e a Temistocle, l'autore conclude questo breve saggio riferendosi direttamente al "Führer der deutschen Geschichte », citato in un suo discorso, e alla situazione tedesca contemporanea. Sempre nel '37, Georg Friedrich Bender discute una tesi su Der Begriff des Staatsmannes bei Thukydides, pubblicata l'anno seguente, e anche questa volta sentiamo parlare della «Einheit von Führer und Volk » che « si fa udire ancora ai giorni d'oggi ${ }^{16}$ ». 
L'affermazione di Tucidide come pensatore della politica è quindi conseguenza della ripresa della "questione tucididea » a partire dallo studio di Schadewaldt secondo le nuove sensibilità politiche contemporanee. Ma proprio questa ripresa del problema compositivo dell'opera di Tucidide, nello stimolare nuovi studi, porterà a restringere sempre di più il campo a cui Schwartz aveva ridato vita ${ }^{17}$. Già nel 1930 un allievo di Regenbogen, August Grosskinsky, aveva presentato come Examenarbeit un lavoro sui capitoli «programmatici » dell'opera dello storico ateniese, in reazione alla tesi di Schadewaldt che vi riscontrava elementi dell'attività iniziale, propriamente storica, di Tucidide. I principi enunciati da Tucidide non si restringono a una parte soltanto dell'opera, né possono avere il senso più delimitato che ad essi si cercava di attribuire, non permettono cioè di creare distinzioni tra i discorsi a partire dalle dichiarazioni "metodologiche» dello storico. L'interpretazione dei passi tucididei da parte di Grosskinsky, nella tesi pubblicata soltanto nel 1936, modifica lo schema più rigido che configurava, secondo Schadewaldt, la conversione di Tucidide alla vera storia e alla riflessione politica ${ }^{18}$. L'anno seguente Harald Patzer (1910-2005), allievo di Jaeger, riprende la questione e ridimensiona ulteriormente la lettura stratigrafica dell'opera di Tucidide (Patzer 1937). Gli studi tucididei di Regenbogen e di Jaeger risuonano ancora attraverso le opere di questi loro allievi. Ancora nel '37, Fritz Bizer studia i capitoli della « archeologia » tucididea - i capitoli dello « storico sofista », secondo Schadewaldt - e li attribuisce, sempre secondo una prospettiva evolutiva, all'attività del Tucidide maturo (come faceva peraltro Jaeger). Dai toni più che mai accesi, Bizer rappresenta Tucidide come un "attischer Imperialist und glühender Bewunderer und Verteidiger des Perikles und seiner Politik». L'archeologia rappresenta una apologia dell'impero pericleo, difeso ad oltranza dallo storico contro gli oppositori, dopo la disfatta (Bizer 1937, p. 41-42). Dall'archeologia ai discorsi, l'attività storiografica del grande storico ateniese, nel suo insieme, viene spiegata per mezzo dell'adesione all'impero e al suo comandante.

19 6. Nello stesso anno in cui era apparso il primo volume di Paideia, Max Pohlenz (1872-1962) intitolava il suo libro su Cicerone e Panezio Antikes Führertum ${ }^{19}$. Pohlenz, nel 1936, riprende le tesi su Tucidide che aveva esposto nei lavori pubblicati alla fine della Prima guerra, per analizzare "la questione tucididea alla luce delle nuove ricerche » (ovvero, i contributi di Wolfgang Schadewaldt, Arnaldo Momigliano, Rose Zahn, August Grosskinsky e Helmut Münch). Pohlenz riconosce l'importanza dell'analisi di Schadewaldt, ma ribadisce, contro la sua tesi, che Tucidide non vuol mai giudicare né essere l'uomo etico normativo («normgebender Ethiker sein ») : egli vuole capire gli avvenimenti politici. La difesa di Tucidide in quanto storico aveva impegnato Pohlenz già in studi elaborati a partire da una recensione al volume di Schwartz nel 1919, ed è da questa posizione, in qualità di studioso della generazione precedente, che recensisce le nuove pubblicazioni su Tucidide. Le critiche a Schadewaldt non impediscono a Pohlenz di soffermarsi sulle posizioni politiche dello storico antico. Tucidide riconosce la crisi etica ateniese dopo la morte di Pericle e il suo significato politico. Il demos, per governare con la democrazia, espelle l'uomo che poteva guidare lo Stato. L'attenzione che dedica Tucidide al timore ateniese dei tiranni verrà giustificata con il sentimento della tragedia del suo popolo (Pohlenz 1936, p. 311). È il conflitto tra lo Stato e i suoi più grandi cittadini a costituire l'oggetto di analisi dello storico, che mostra la presenza di questo conflitto sin dalle guerre persiane, con Temistocle. Il generale ateniese non rappresenta però un paradigma (questa era la posizione di Arnaldo Momigliano, in 
precedenza discussa da Pohlenz), ma "piuttosto il primo segnale di uno sviluppo inarrestabile » (p. 312, corsivo mio).

Questi temi sono sinteticamente ripresi, nel 1938, da Helmut Berve (1896-1979), il quale pubblica un breve volume su Tucidide nella serie "auf dem Wege zum nationalpolitischen Gymnasium ». Significativamente, questo volume è suddiviso in capitoli intitolati : «Der Forscher », «Der Künstler» e, per ultimo, "Der politische Denker». Berve avverte il rischio di inserire Tucidide tra i «filosofi», laddove « Denker, Künstler und Forscher eines sind » (p. 40). Con tale precisazione, l'autore può riprendere il risultato delle ricerche precedenti : è come pensatore politico che Tucidide scopre la regolarità dell'accadere storico. Con Regenbogen, Berve afferma che Tucidide « durch politische Erkenntnis der politischen Praxis dienen wollte» (p. 45). Dal dialogo dei Melii l'autore passa direttamente al Mein Kampf hitleriano, nel richiamo alla "Sicherheit, Ehre und Nützen des Staates ${ }^{20} »$ (p. 49). Con Tucidide si scopre la « Ratio aller Politik». Ma il singolo uomo politico svolge un ruolo secondario nell'opera dello storico : per Tucidide l'individualità è rappresentata dalla " comunità statale ». Le due forze irrazionali della storia che emergeranno nelle vicende successive delle città greche - l'arbitrio dell'azione individuale e la tyche - non trovano spazio nell'opera tucididea e nell'Atene a lui contemporanea.

21 7. Della novità rappresentata dal nuovo quadro degli studi tucididei vi era consapevolezza, come rivela uno studioso della « vecchia » generazione, Alfred Körte (1866-1946), in una recensione pubblicata su Gnomon nel 1944 :

In dem vielstimmigen Chor der neuen Thukydidesforscher lassen sich zwei Richtungen scheiden: die einen stellen den Denker und Künstler, die andern den Historiker voran (Körte 1944, p. 189-190).

Oggetto della recensione di Körte sono i saggi curati da Helmut Berve per il primo volume su Das neue Bild der Antike, apparso nel '42, e, nel caso particolare, il saggio di Franz Egermann (1905-1989) su Tucidide ${ }^{21}$. Secondo l'anziano Körte, Egermann è tra coloro che leggono Tucidide in quanto storico (e giustamente, s'intende).

Egermann, a sua volta, si era confrontato già prima con i nuovi studi tucididei. Nel 1937 aveva recensito criticamente i lavori di Grosskinsky e di Patzer: per mezzo dei discorsi, frammezzati alla narrazione storiografica, e delle considerazioni metodologiche inserite da Tucidide all'inizio dell'opera si correva il rischio di creare l'immagine di un Tucidide "politico» e "artista», piuttosto che «storico». Egermann si mostra preoccupato con analisi che riscontrano, nei discorsi, "Zeugnisse eines normativ korrigierenden Staatsethikers, nicht eines Historikers » (p. 1480). In realtà, anche Patzer aveva avvertito che Tucidide «nichts Staatstheoretiker ist und nicht Soziologe, sondern Historiker » (Patzer 1937, p. 101). Nel quadro mutato dell'analisi del processo compositivo tucidideo, resta sempre presente il richiamo agli schemi interpretativi di Schadewaldt.

Franz Egermann recensisce anche il volume di Berve su Tucidide per Gnomon, nel 1941. Egermann concede all'autore : « Tucidide è un pensatore assai profondo... paragonabile a Eschilo ». Ma non accetta, perciò, che Tucidide non riconosca la "Persönlichkeit als geschichtsbildende Kraft ». Gli esempi di Temistocle e di Pericle gli sono di aiuto, nel contrapporsi al riferimento di Berve alla « Kollektivpersönlichkeit des Gemeinwesens ». Si sente, nei temi di Berve e del suo recensore, l'eco degli studi che, durante gli anni '30, hanno cercato di offrire un nuovo Bild dello storico di Atene. Egermann non riscontra in Tucidide un interesse prevalente per le forme di rappresentazione collettiva degli 
attori storici. In questo risiede, riafferma l'autore nel saggio pubblicato nel 1942, la natura propriamente storica dell'opera di Tucidide : lo storico si fa vedere in quanto tale nell'attenzione che dedica all'individuo superiore. Sono lontani i tempi in cui sembrava mancare il vero ethos alla nazione e ai suoi governanti. «Der wahrhaft Verursachende geschichtlichen Geschehens ist der überragende Mann ${ }^{22} »$ (Egermann 1942, p. 500).

1938 Berve, Thukydides; Nestle, Der Friedensgedanke in der Antiken Welt; Weinstock (a cura di), Thukydides, Der Grosse Krieg.

1939 Luschnat, Die Feldherrnreden im Geschichtswerk des Thukydides (ed.1942).

1940 Gundert, "Athen und Sparta in den Reden des Thukydides".

1941 Egermann, rec. a Berve (1938).

1942 Egermann, Die Geschichtsbetrachtung des Thukydides.

1943 Reinhardt, Thukydides und Machiavelli.

1944 Körte, rec. a Berve/Egermann (1942); Regenbogen (a cura di), Thukydides, Politische Reden (ed. 1949).

\section{BIBLIOGRAFIA}

BENDER, G. F. (1937), Der Begriff des Staatsmannes bei Thukydides, Konrad Triltsch, Würzburg.

BERVE, H. (1938), Thukydides, Diesterweg, Frankfurt a.M.

BERVE, H. (a cura di) (1942), Das neue Bild der Antike, I, Koehler \& Amelang, Leipzig.

BIZER, F. (1937), Untersuchungen zur Archäologie des Thukydides, Tübingen (rist. Wissenschaftliche Buchgesellschaft, Darmstadt 1968).

BOGNER, H. (1937), Thukydides und das Wesen der altgriechischen Geschichtsschreibung, Hanseatische Verlagsanstalt, Hamburg. 
CALDER III, W. M. (a cura di) (1992), Werner Jaeger Reconsidered (Illinois Classical Studies, Supplement 3), Scholar Press, Atlanta.

CANFORA, L. (1980), Ideologie del classicismo, Einaudi, Torino.

CHANIOTIS, A. \& THALER, U. (2006), Altertumswissenschaft, in Die Universität Heidelberg im Nationalsozialismus, a cura di W. V. Eckhardt, V. Sellin \& E. Wolgast, Springer, Heidelberg.

DALFEN, J. (1990), “Franz Egermann”, Gnomon 62, p. 755-757.

DIETZFELBINGER, E. (1934), Thukydides als politischer Denker. Inaugural-Dissertation, Karl Döres, Erlangen.

EGERMANN, F. (1937), “Neue Forschungen zu Thukydides”, Deutsche Literaturzeitung 58, p. $1471-1480 ; 1505-1509$.

EGERMANN, F. (1941), recensione a Berve (1938), Gnomon 17, p. 203-207.

EGERMANN, F. (1942), Die Geschichtsbetrachtung des Thukydides, in Berve (1942), p. 272-302.

FINLEY Jr., J. H. (1939), recensione a H. Patzer, American Journal of Philology 60, p. 108-111.

FINLEY Jr., J. H. (1940), recensione a G. F. Bender, American Journal of Philology 61, p. 249.

FLASHAR, H. (2005), Biographische Momente in schwerer Zeit, in Wolfgang Schadewaldt und die Gräzistik des 20. Jahrhunderts, a cura di T. Szlezák, Olms, Hildesheim, p. 151-169.

GROSSKINSKY, A. (1936), Das Programm des Thukydides, Junker und Dunnhaupt, Berlin.

GUNDERT, H. (1940), “Athen und Sparta in den Reden des Thukydides”, Antike 16, 98-114 (=

Thukydides, "Wege der Forschung", Darmstadt 1968, p. 114-134).

GUNDERT, H. (1967), “Otto Regenbogen”, Gnomon, 39, p. 219-221.

HOFFMANN, Chr. (1988), Juden und Judentum im Werk deutscher Althistoriker des 19. und 20.

Jahrhunderts, Brill, Leiden.

JACOBY, F. (1930), recensione a Schadewaldt (1929), Historische Zeitschrift 142, p. 324-328 (=

Abhandlungen zur griechischen Geschichtschreibung, a cura di H. Bloch, Brill, Leiden 1956, p. 239-242).

JAEGER, W. (1924), Die griechische Staatsethik im Zeitalter des Plato, in Humanistische Reden und Vorträge, zweite erweiterte Auflage, De Gruyter, Berlin 1960, p. 87-102.

JAEGER, W. (1933), “Die Erziehung des politischen Menschen und die Antike”, Das Volk im Werden I, p. 43-49.

JAEGER, W. (1934), Paideia. Die Formung des griechischen Menschen. Erst Band, Walter de Gruyter, Berlin und Leipzig, prima ed. 1934, sec. ed. 1936 (cit. anche dalla trad. it. di L. Emery e A. Setti, La Nuova Italia, Firenze 1967).

KÖRTE, А. (1944), recensione a Berve (1942), Gnomon 20, p. 177-192.

LUSCHNAT, O. (1942), Die Feldherrnreden im Geschichtswerk des Thukydides (Philologus Supplementband 34), Leipzig.

MALITZ, J. (1998), Römertum im « Dritten Reich »: Hans Oppermann, in Imperium Romanum. Festschrift für Karl Christ, Franz Steiner, Leipzig, p. 519-543.

MEINECKE, F. (1924), Die Idee der Staatsräson in der neueren Geschichte, R. Oldenbourg, MünchenBerlin. 
MOMIGLIANO, A. (1959), recensione a H. Berve, Storia greca, in Rivista Storica Italiana 71, p. 665-672; e in Terzo contributo alla storia degli studi classici e del mondo antico, Edizioni di Storia e Letteratura, Roma 1966, p. 699-708.

MÜNCH, H. (1935), Studien zu den Exkursen des Thukydides, in Quellen und Studien zur Geschichte und Kultur des Altertums und des Mittelalters, Reihe D, heft 3, im Selbstverlag von F. Bilabel, Heidelberg. NÄF, B. (1986), Von Perikles zu Hitler? Die athenische Demokratie und die deutsche Althistorie bis 1945, Peter Lang, Bern - Frankfurt am Main - New York.

NESTLE, W. (1934), “Thukydides als politischer Erzieher”, Unterricht und Forschung, p. 157-167.

NESTLE, W. (1938), Der Friedensgedanke in der Antiken Welt (Philologus, Supplementband 31), Dieterich'sche Verlag, Leipzig.

NESTLE, W. (1965), Bibliographie Wilhelm Nestle, zu seinem 100. Geburtstag, a cura di Rudolf Nestle, Stuttgart.

OPPERMANN, H. (1935), “Altertumswissenschaft und politische Erziehung”, Neue Jahrbücher für Wissenschaft und Jugendbildung 11, p. 367-372.

PATZER, H. (1937), Das Problem der Geschichtsschreibung des Thukydides und die thukydideische Frage, Junker und Dünnhaupt, Berlin.

POHLENZ, M. (1919), “Thukydidesstudien I”, Nachrichten der Gesellschaft der Wissenschaften zu Göttingen, p. 95-138 (= Kleine Schriften, II, a cura di H. Dörrie, rist. Olms, Hildesheim 1965, p. 210-253).

POHLENZ, M. (1920), “Thukydidesstudien II”, Nachrichten der Gesellschaft der Wissenschaften zu Göttingen, p. 56-68; 68-82 (= Kleine Schriften, II, a cura di H. Dörrie, rist. Olms, Hildesheim 1965, p. 254-280).

POHLENZ, M. (1934), Antikes Führertum. Cicero De officiis und das Lebensideal des Panaitios, Teubner, Leipzig-Berlin.

POHLENZ, M. (1936), “Die thukydideische Frage im Lichte der neueren Forschung”, Göttingische Gelehrte Anzeigen 198, p. 281-300 (cit. da Kleine Schriften, II, a cura di H. Dörrie, rist. Olms, Hildesheim 1965, p. 294-313;= Thukydides, “Wege der Forschung”, Darmstadt 1968, p. 59-81).

REBENICH, S. (2001), “Alte Geschichte in Demokratie und Diktatur: Der Fall Helmut Berve”, Chiron 31, p. 457-496.

REGENBOGEN, O. (1930), "Drei Thukydidesinterpretationen", Monatschrift für Höhere Schulen, Berlin, p. 21-29 (= Kleine Schriften, Beck, München 1961, p. 206-216= H. Herter, a cura di, Thukydides, "Wege der Forschung", Darmstadt 1968, p. 10-22).

REGENBOGEN, O. (1933), “Thukydides als politischer Denker”, Gymnasium 44, p. 2-25

(= H. Herter, a cura di, Thukydides, "Wege der Forschung", Darmstadt 1968, p. 23-58= Kleine Schriften, Beck, München 1961, p. 217-247).

REGEnBogen, O. (1934), "Das Altertum und die Politische Erziehung”, Neue Jahrbücher für Wissenschaft und Jugendbildung 10, p. 211-225.

REGENBOGEN, O. (a cura di) (1949), Thukydides. Politische Reden, Koehler \& Amelang, Leipzig.

REHM, A. (1934), “Die sizilischen Bücher des Thukydides”, Philologus 89, p. 133-160. 
REINHARDT, K. (1966), Vermächtnis der Antike. Gesammelte Essays zur Philosophie und Geschichtsschreibung, hrsg. v. C. Becker, Zweite, durchgesehene und erweiterte Auflage, Vandenhoeck \& Ruprecht, Göttingen.

SCHADEWALDT, W. (1929), Die Geschichtsschreibung des Thukydides, Weidmannsche Buchhandlung, Berlin.

SCHADEWALDT, W. (1934), “Einzelner und Staat im politischen Denken der Griechen”, Vergangenheit und Gegenwart 24, p. 16-32.

SCHWARTZ, E. (1919), Das Geschichtswerk des Thukydides, Dritte, Unveränderte Auflage 1929, rist. Georg Olms, Hildesheim 1969.

SKUTSCH, O. (1992), "Recollections of Scholars I have known", a cura di A. Bierl e W. M. Calder III, Harvard Studies in Classical Philology 94, p. 387-408.

STAHL, H.-P. (1966), Thukydides. Die Stellung des Menschen im geschichtlichen Prozeß, Beck, München (trad. ingl. The Classical Press of Wales 2003).

Strasburger, H. (1936), “Zu Thukydides 6, 15”, Philologus 91, p. 137-152.

WASSERMANN, F. (1931), “Das neue Thukydidesbild”, Neue Jahrbücher für Wissenschaft und

Jugendbildung 7, p. 248-258.

WEINSTOCK, H. (1934), Polis. Der Griechische Beitrag zu einer deutschen Bildung heute an Thukydides erläutert, Die Runde, Berlin.

WEINSTOCK, H. (a cura di) (1938), Thukydides, Der Grosse Kriege, Alfred Kröner Verlag, Stuttgart.

WESSELING, K.G. (2001), “Werner Jaeger”, in Biographisch-Bibliographisches Kirchenlexikon, XVIII, coll. 717-749.

ZAHN, R. (1934), Die erste Periklesrede (diss. Kiel 1933), Noske, Borna-Leipzig 1934.

ZIEGLER, K. (1929), “Der Ursprung der Exkurse im Thukydides”, Rheinisches Museum 78, p. 58-67.

\section{NOTE}

1. L' interesse per il Tucidide politico negli anni '30 è stato notato da Stahl (1966), p. 24-25, e da Näf (1986), p. 193-196. Ma di questo vi era già consapevolezza tra gli stessi studiosi del periodo: si veda, ad esempio, Berve (1938), p. 40, e Körte (1944), p. 189-190, menzionati infra.

2. Jaeger (1924). Nel testo di Jaeger si trovano riferimenti a Tucidide e al dialogo dei Melii, alla «ragion di Stato» e allo spuntare del «machiavellismo», temi ugualmente richiamati da Meinecke nel volume sulla Idea di Ragion di Stato pubblicato in quell'anno (Meinecke 1924). Il saggio di Jaeger verrà significativamente ripubblicato sulla sua rivita «Die Antike» nel 1934. Su Jaeger cfr. Näf (1986), p. 187-191, e Wesseling (2001), nonché i saggi contenuti in Calder (1992), in particolare, per i temi qui trattati, i saggi di M.Chambers, B. Näf e D. O. White. Sull'analisi di Tucidide in Paideia vedi infra.

3. Si noti che il volume di Schwartz, Das Geschichtswerk des Thukydides, viene ristampato, con una breve nota introduttiva dell'autore, nel 1929.

4. Nel 1934 Schadewaldt ritorna alle questioni di politica e di etica statale studiando «l'individuo e lo Stato nel pensiero politico greco» (Schadewaldt 1934). Per le attività di Schadewaldt durante gli anni del nazismo - e per i suoi rapporti a Friburgo con Martin Heidegger - si veda Flashar (2005). 
5. Cfr. Jacoby (1930). L'interesse di Jacoby per la ripresa della questione tucididea si manifesta attraverso la tesi della sua allieva, Rose Zahn, su Die erste Periklesrede, pubblicata, con l'aggiunta di note di Jacoby, nel 1934 (cfr. Zahn 1934; Pohlenz 1936).

6. Gli Jahrbücher costituiscono la continuazione dei Neue Jahrbücher für das Klassische Altertum, Geschichte und Deutsche Literatur (o für Pädagogik), che fino al '29 erano stati diretti da Johannes Ilberg, storico della medicina, e che già nella Prima guerra e negli anni successivi avevano ospitato varie riflessioni sulla storiografia tucididea e i suoi richiami storici attuali. La pubblicazione degli annali (con il nuovo titolo dal 1925) passa nel 1930 sotto la direzione di Heinrich Weinstock (su cui vedi infra). Lo spazio dedicato all'antichità classica sembra ora ridimensionato, anche se mai irrilevante.

7. Al contrario, in quegli anni un allievo di Regenbogen, August Grosskinsky, preparava un lavoro di tesi che almeno parzialmente era rivolto contro l'analisi di Schadewaldt (infra).

8. Nell'attenzione di Regenbogen per i discorsi - e per il pathos della sconfitta - si sente l'eco dell'articolo, non citato, di Wassermann. Regenbogen non perderà, comunque, questo interesse particolare per i discorsi, preparando, negli anni della guerra, una traduzione dei «discorsi politici» tucididei, consegnata all'editore nel 1944, poi pubblicata nel 1949, con una nota del ' $46 \mathrm{e}$ con una versione modificata e semplificata dell'articolo del '33 (in cui significativamente si aggiunge al Tucidide politico il Tucidide «militare») (Regenbogen 1949). Sulla scia di Regenbogen, il giovane Hermann Gundert pubblica su Die Antike, nel 1940, un saggio sui discorsi degli Spartani e Ateniesi in Tucidide (Gundert 1940). Gundert scriverà poi la nota biografica su Regenbogen, per Gnomon, alla morte dello studioso (Gundert 1967).

9. Ancora nel '34, Regenbogen parla di Römische Heldentaten sulla rivista «Deutsche Wille». In quell'anno sceglie i Dori come argomento del suo corso; l'anno seguente, si occupa di «Omero e i Tedeschi». Nel '37, Regenbogen viene allontanato dall'insegnamento, a causa degli antenati ebrei della moglie. Su Regenbogen cfr. Gundert (1967); Näf (1986), p. 193-194; e soprattutto Chaniotis \& Thaler (2006). Sulla sua sospensione dal lavoro - e quindi la sua non inclusione nell'elenco dei docenti proposti alla sostituzione di Jaeger a Berlino -, si veda Flashar (2005), p. 163.

10. Jaeger (1934), cap. VI (Thukydides als politischer Denker), p. 479-513. Su Jaeger e Tucidide si veda in particolare il saggio di M. Chambers, in Calder (1992), p. 25-35, il quale però utilizza una ristampa di Paideia e non può quindi notare la modifica operata dall'autore dopo la prima edizione.

11. Jaeger (1933), citato da Chambers, in Calder (1992), p. 33.

12. Si tratta della rivista Unterricht und Forschung, dal sottotitolo Wissenschaftliche Zeitung auf nationalsozialistischer Grundlage (Nestle 1934). Sempre nel '34, Nestle pubblica una monografia su Menschliche Existenz und politische Erziehung in der Tragödie des Aischylos, a riprova della rilevanza attuale del tema della educazione politica. Per la bibliografia dell'autore si veda Nestle (1965). Sempre per Unterricht und Forschung Nestle analizza, nel 1935, «Die Juden in der griechischrömischen Welt» (Hoffmann 1988, p. 266). Già nel '39 si dedicherà alla riflessione antica sulla pace (Nestle 1939), in contrapposizione a chi voleva che nel mondo antico la pace rappresentasse un'eccezione all'interno di un continuo stato di guerra. Questo Nestle «pacifista» con gli antichi conclude, però, omericamente, esaltando l'amynesthai peri patres.

13. Nel ' 54 Regenbogen pubblica su Gnomon (Bd. 26, p. 289-299) una dura recensione a un volume di Weinstock sulla «tragedia dell'Umanesimo», pubblicato l'anno precedente.

14. L'argomento di Rehm, i libri siciliani, si pone naturalmente in diretta relazione con la tesi di Schadewaldt. Così anche Hermann Strasburger tratterà, nel '36, dell'interpretazione di un passo del sesto libro (Strasburger 1936).

15. Su Bogner cfr. Näf (1986), p. 129-135 (con bibliografia)墭; Malitz (1998), p. 526 s. Nel'36 Bogner aveva recensito criticamente il volume Polis, di H. Weinstock, per Gnomon (p. 215-217).

16. Cfr. Bender (1938), che cito a partire dalla recensione di Finley (1940), p. 249.

17. Così viene notato già da Finley (1939). 
18. Anche un altro allievo di Regenbogen, Helmut Münch, si dedicò a Tucidide, pubblicando, nel '35, a Heidelberg, la sua dissertazione sugli excursus tucididei (Münch 1935; cfr. Pohlenz 1936). Münch si mostra fedele allo schema di Schadewaldt, che contrappone al saggio precedente di K. Ziegler sugli excursus (Ziegler 1929). Si vedano le osservazioni di Münch sul lavoro di Grosskinsky non ancora pubblicato (p. 19 n. 3).

19. Pohlenz (1934). Cfr. la critica a Pohlenz di Hans Oppermann, il quale si domanda se è giusto applicare all'ideale di Panezio un termine - Führer - che «ha per noi un contenuto del tutto concreto, definito nella realtà politica»: l'antica Führertum va osservata piuttosto in Solone, Pericle, Alessandro, Cesare e Augusto (Oppermann 1935). Già dal '34 Oppermann, che era stato allievo di Regenbogen, pubblica sui Neue Jahrbücher für Wissenschaft und Jugendbildung resoconti di lavori sull'antichità (sull'attività di Oppermann durante il periodo nazista cfr. Malitz 1998; si veda anche Chaniotis \& Thaler 2006, p. 393, con ulteriore bibliografia). Nel 1933 erano stati pubblicati nei Neue Jahrbücher (p. 572-574) i «Leitsätze des Vereins Bayerischen Philologen», ben corredati da citazioni dal Mein Kämpf, con riferimenti agli esempi di «politische Führertum» presso gli antichi; e questi Führer ci parlano, in parte, attraverso le opere di Tucidide e di Cesare. Su Pohlenz e la Führertum si veda Canfora (1980), p. 133 s.

20. Questa citazione di Hitler è stata ricordata da Momigliano (1959). Sulla figura di Berve si veda Rebenich (2001), con ampia bibliografia.

21. Su Egermann cfr. Dalfen (1990). Per i suoi rapporti con Jaeger si veda la breve menzione in Skutsch (1992), p. 394.

22. È necessario menzionare, in conclusione a questa serie di studi tedeschi su Tucidide, il saggio di Karl Reinhardt su Thukydides und Machiavelli (Reinhardt 1966, p. 184-217). Risultato di una conferenza presentata il 3 febbraio 1943 a Berlino, nella serie di Studia Humanitatis, curata da Ernesto Grassi, il testo doveva essere incluso nel terzo volume di Geistigen Überlieferungen, mai pubblicato. Una successiva «Sonderausgabe», prevista in Italia, non è mai stata completata. Dedicandosi a Tucidide negli anni finali della Seconda guerra, Reinhardt osserva criticamente l'analisi di Eduard Schwartz, che di Tucidide si era occupato negli anni finali del conflitto precedente. Allo stesso tempo, nel riprendere il confronto con Machiavelli, Reinhardt si riallaccia a una discussione che era stata particolarmente intensa negli anni della Prima guerra e che aveva coinvolto i principali intellettuali tedeschi, tra Max Weber e Friedrich Meinecke. Alla contrapposizione tra politica (di potenza) e morale (etica statale), la lettura di Reinhardt aggiunge nuovi elementi interpretativi - il «silenzio» di Tucidide, «l'umano» - meno consoni agli studi sul Tucidide «politico», e rivolge l'attenzione a momenti narrativi - la peste, Melo, Micalesso - in cui il pathos antico e il sentimento moderno della distruzione si confondono. A questi temi, che richiamano un approfondimento che va oltre lo scopo di questo saggio, sarà opportuno tornare in uno studio più ampio.

\section{AUTORE}

\section{PAULO BUTTI DE LIMA}

butti@lettere.uniba.it 